\title{
ESEL UND RINDER. MYTHOLOGISCHE PARADIGMATA IM SCHLUßTEIL DER METAMORPHOSEN DES APULEIUS ${ }^{1}$
}

\author{
Thomas Gärtner
}

\begin{abstract}
In der vorliegenden Arbeit werden zwei Partien aus den Metamorphosen des Apuleius näher betrachtet, in denen eine Verbindung zwischen „Eseln und Rindern“ hergestellt wird: An der ersten Stelle wird der sodomistische Geschlechtsverkehr des vereselten Lucius mit einer menschlichen Frau explizit in Verbindung gebracht mit der mythischen Pasiphae-Geschichte; an der zweiten scheint eine bislang verkannte strukturelle Parallele zwischen Lucius und der ovidischen Isis-Erzählung vorzuliegen. An den beiden behandelten Beispielen lässt sich paradigmatisch das weite Spektrum intertextueller Berührung zwischen offen ausgesprochenem Vergleich und verdeckter sinntragender Anpielung erkennen.
\end{abstract}

1. Eine explizite Parallele: Der Esel Lukios bzw. Lucius und der Stier der Pasiphae im griechischen Eselsroman und bei Apuleius.

Sowohl in den Metamorphosen des Apuleius als auch in deren griechischem Vorbild, das aus dem unter den Werken Lukians erhaltenen Onos zu rekonstruieren ist, zeichnet sich der durch ein magisches Mißgeschick in einen Esel verwandelte Lukios bzw. Lucius aus durch eine Kombination von äußerlicher Eselgestalt und inneren menschlichen Eigenschaften. In der Schlussphase seiner Irrfahrten wird der menschliche Teil seines Wesens entlarvt, insofern sich der gefräßige Esel während seines Aufenthalts in einer Bäckerei nicht mehr auf eseltypische Nahrung beschränkt. Damit beginnt die letzte Phase seiner Leiden, die sich in seiner Ausstellung als ein zirkus-artiges Schauobjekt konkretisieren. Seine menschlichen Eigenschaften werden aber nicht nur zu solcher kulinarischer Exhibition missbraucht, sondern er muß außerdem auch noch die sodomistischen Triebe einer vornehmen reichen Frau befriedigen. ${ }^{2}$ In der letzten Szene vor seiner Zurückverwandlung werden diese beiden Aspekte (einerseits Exhibition, andererseits Befriedigung weiblicher Triebe) kombiniert: Lucius soll öffentlich bei einer Volksbelustigung mit einer zweiten Frau, einer verurteilten Schwerverbrecherin Geschlechtsverkehr ausüben. ${ }^{3}$

\footnotetext{
${ }^{1}$ Zur Bedeutung der mythologischen Paradigmata Ganymed und Pegasus in Hinsicht auf die „ideologische Einheit und teleologische Ausrichtung des Romans" vgl. Babo 2000. Speziell zum Einfluß der ovidischen Metamorphosen auf den Roman des Apuleius vgl. Müller-Reineke 2000, insbesondere zu den sodomistischen Szenen am Ende des zehnten Buchs 190-193. Noch nicht zugänglich war mir Hindermann 2009.

${ }^{2}$ Effe 1976, bes. 372 f., erkennt hier speziell eine „obszön-parodistische Umgestaltung der Melite-KleitophonHandlung bei Achilleus Tatios".

${ }^{3}$ Zum Höhepunkt von Lucius' Qualen am Ende des zehnten Apuleius Buchs vgl. Scobie 1978, bes. 54, wo Buch X als „nadir of crime and perversion” bezeichnet wird: „Lucius' reward for gratifying the bestial vesana libido of the Corinthian woman is to be exposed ad bestias to enact a Pasiphae mime with the perpetrator of a multiforme scelus".
} 
In dieser Szene weicht bekanntlich Apuleius in entscheidender Weise von seiner griechischen Vorlage ab: ${ }^{4}$ Sein Lucius nimmt in dem Moment, als ihm die Verbrecherin zugeführt wird, Reißaus aus der Menschenwelt und wendet sich schließlich in einem verzweifelten Gebet an Isis um Hilfe; nach seiner schließlichen Erlösung und Zurückverwandlung bei einem Isis-Fest ändert er sein Leben und wird zum Mysten.

Dagegen findet der Lukios des griechischen Originals in derselben Szene im letzten Moment die rettenden Rosen ohne göttliche Hilfe, wird so noch innerhalb der Vorführung in einen Menschen zurückverwandelt, hat sich dann noch gegen den Vorwurf der schwarzen Magie zu verteidigen und kehrt schließlich, hiervon freigesprochen, mit seinem Bruder in seine Heimat zurück. Zuvor stattet er jedoch der reichen Dame, mit der er zuvor in Eselsgestalt mehrfach Beischlaf hatte, noch einen Besuch mit eindeutiger Absicht ab, wird von dieser aber schließlich nackt vor die Tür gesetzt, da er in menschlicher Gestalt enttäuschenderweise ihren Erwartungen nicht mehr gerecht wird.

Der konzeptionell bei Apuleius völlig veränderte Schlußteil legt den Gedanken nahe, dass Apuleius auch die sodomistische Beischlafszene ${ }^{5}$ mit der reichen Frau vor der Rückverwandlung gegenüber seinem griechischen Vorbild nicht ganz unverändert gelassen haben wird: Denn während diese Szene im griechischen Original vor allem

\footnotetext{
${ }^{4}$ Abweichend von dieser communis opinio Bohm 1973 (Vertreter ähnlicher Thesen und weitere Doxographie bei Schlam \& Finkelpearl 2000: 36-41, besonders 38). Bohm nimmt an, das griechische Original habe am Ende einen scherzhaft ironischen religiösen Schluß gehabt, der in der uns vorliegenden Epitome ausgespart, von Apuleius dagegen ernstgenommen worden sei (freilich sollte man doch dem Apuleius a priori eine wesentlich größere gestalterische Freiheit im Umgang mit seinem griechischen Original zugestehen als einem griechischen Epitomator). Übrigens tendiert die moderne Apuleius-Forschung dazu, gerade auch im religiös geprägten Schlußbuch des Apuleius humoristisch parodistische Züge zu sehen, vgl. insbesondere Murgatroyd 2004, der selbst diese Auffassung vertritt und verschiedene Vorgänger nennt, ferner die eher neutral resümierende Arbeit von Töchterle (1998); Harrison (2000: 248) sieht Buch XI sogar als „an amusing satire on religious mania and youthful gullibility". Daß der in der Antike sprichwörtlich potente Esel, der vor der Verrichtung seiner öffentlichen „Arbeit” am Ende von Buch X Reißaus nimmt und in eine religiöse Welt kultischer Reinheit flieht, komische Züge trägt, wird man nicht bestreiten. - In diametralem Gegensatz zu Bohm schreibt übrigens der Patriarch Photios im 129. Kapitel seiner Bibliotheke gerade dem Original des griechischen Eselsromans, dem dubiosen „Lukios von Patrai”, gegenüber der ironisierenden Tendenz der angeblich lukianischen Epitome tendenzielle Ernsthaftigkeit zu (zur Zweifelhaftigkeit dieses Urteils vgl. Kussl 1990). - Ein exaktes Gegenstück zu der These Bohms, der den Schluß von Apuleius' Metamorphosen in die griechische Urfassung zurückprojiziert, liefert van Mal-Maeder 1997 (in engem Anschluß hieran jetzt N. Holzberg in der neuesten Auflage von Der antike Roman, 2006: 110 f.): Das Ende von Apuleius' Roman sei verloren, Lucius habe sich vom Isiskult wieder abgewandt (so daß sein Mystendasein im Schlußbuch nur eine von Apuleius eingeschobene Episode wäre), sei nach Korinth zu seiner Liebhaberin, mit der er als Esel Umgang hatte, zurückgekehrt und sei schließlich von ihr (wie in der pseudo-lukianischen Fassung und aller Wahrscheinlichkeit nach auch im griechischen Original) gedemütigt worden. ${ }^{5}$ Die Einleitung der Szene mit den Worten et iam dudum praestolantem cubiculo meo matronam offendimus (20.1) hat übrigens in der griechischen Romanliteratur eine noch engere Parallele als die Epitome des grie-

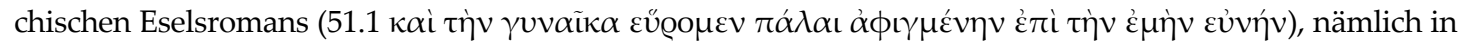

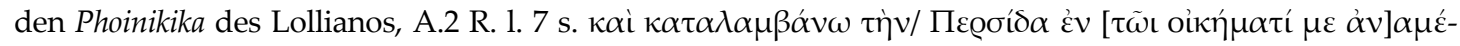
vov $\sigma \alpha v$ (p. 334 Stephens/Winkler). Auch in diesem Fragment wird ein Ich-Sprecher eine ganze Nacht lang von einer reichen, mit Gefolgschaft auftretenden Frau sexuell ausgebeutet, die ebenfalls Geld bezahlt für die ihr erbrachte Leistung. Insofern sich bei Lollianos die Zahlung an den Sprecher selbst richtet (zuerst soll sie in Form goldener Schmuckteile erfolgen, welche die Dame vor der Verrichtung ablegt, dann soll, nachdem der Sprecher sich weigert, den Schmuck anzunehmen, ein Sklave die Auszahlung in bar vornehmen), nicht wie bei Apuleius an den Halter eines Esels, ist es klar, dass man bei Lollianos keinen in ein Tier verwandelten Sprecher vor sich hat. Die Parallele tritt neben andere auffällige Gemeinsamkeiten zwischen Lollianos und Apuleius, die auf die Tatsache führen, "that what the ass narrates in Apuleius seems in Lollianos to be communicated by a human narrator" (Stephens/Winkler 322-325, Zitat von S. 324).
} 
im Kontrast mit der Schlußszene, dem vergeblichen Anlauf des Lukios in menschlicher Gestalt, wirken soll, ${ }^{6}$ muß die entsprechende Beischlafszene bei Apuleius ihre Wirkung ohne eine solche Kontrastszene ausüben. Und in der Tat lassen sich bei Apuleius trotz aller sprachlichen und sachlichen Übernahmen deutlich veränderte Tendenzen nachweisen, die in der verschiedenen Applikation des mythischen Vorbilds der Pasiphae kulminieren.

Zunächst unterscheidet sich die Art, wie der Esel Lucius das ihn stimulierende Verhalten der reichen Frau bewertet: Der Esel in der griechischen Version hebt hervor, dass diese ihn wie einen menschlichen Liebhaber behandelt (51.5 عĩ $\alpha \dot{\alpha} \mu \varepsilon \kappa \alpha \tau \varepsilon \phi i ́ \lambda \eta \sigma \varepsilon$

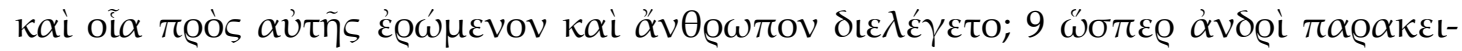

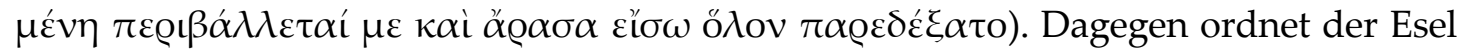
des Apuleius das Verhalten der Frau unter als typisch empfundene weibliche Verhaltensweisen ein (10.21.2 tunc exosculata pressule, non qualia in lupanari solent basiola iactari vel meretricum poscinummia vel adventorum negantinummia, sed pura atque sincera instruit et blandissimos adfatus; 3 et cetera, quis mulieres et alios inducunt et suas testantur adfectationes); dabei wird die Absurdität des weiblichen Verhaltens auch noch zusätzlich gekennzeichnet (ohne Parallelen in der griechischen Epitome) durch die wörtlichen Wiedergaben von grotesken an den Esel gerichteten Koseworten (21.3 "amo" et "cupio" et "te solum diligo" et "sine te iam vivere nequeo" et cetera; 22.3 "teneo te" inquit "teneo, meum palumbulum, meum passerem"). Während also der Esel des griechischen Originals es nur als auffällig empfindet, dass er wie ein Mensch behandelt wird, ordnet derjenige des Apuleius die grotesken Verhaltensweisen der reichen Frau in ein ziemlich anrüchiges Gesamtbild weiblicher Erotik ein.

Ein weiterer Unterschied besteht im Grad der Zuversicht des Esels bezüglich seiner eigenen Potenz: Im griechischen Text wird aus der langdauernden Abstinenz des Lukios (der nicht einmal eine Eselin als Partnerin hatte, geschweige denn eine menschli-

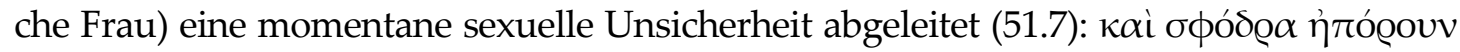

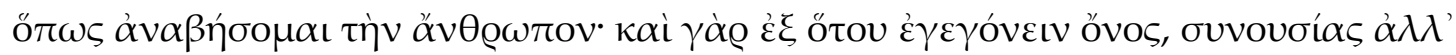

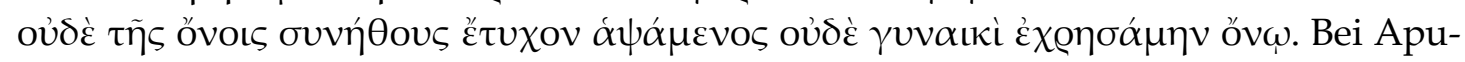
leius wird dagegen gerade aus dieser langen Abstinenzzeit die besondere Fähigkeit des Esels abgeleitet (21.4): quippe cum nil novi nihilque difficile facturus mihi viderer, praesertim post tantum temporis tam formosae mulieris cupientis amplexus obiturus. Die Furcht, die zarte Frau durch seine grobschlächtigen Glieder zu verletzen, tritt im Griechischen

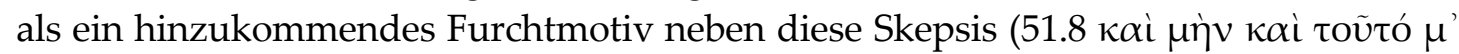

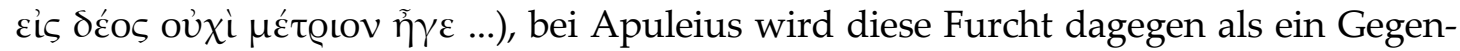
satz an die Zuversicht des Esels angeschlossen (22.1 sed angebar plane non exili metu reputans ...).

Im Griechischen leitet die sexuelle Unsicherheit des Esels weiter über zu einer ob-

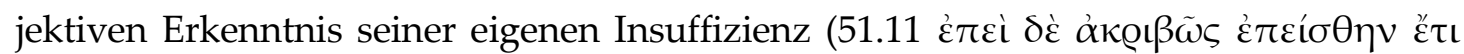

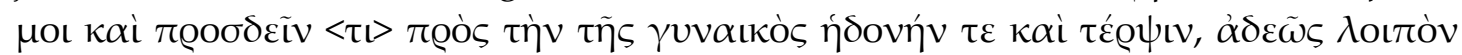

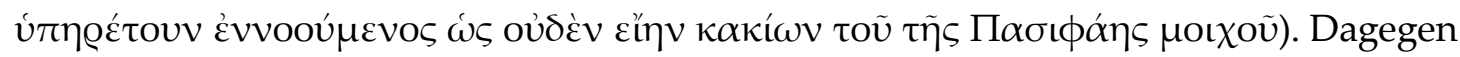
erkennt der Esel des Apuleius seine eigene Insuffizienz nicht etwa als ein objektives Faktum, sondern gelangt nur durch die stimulierenden Bemühungen der Frau zu dem

\footnotetext{
${ }^{6}$ Vgl. Bernsdorff 1997, besonders 35-37. Bernsdorff betont insbesondere zurecht, dass in der ersten Szene die Frau, in der zweiten dagegen Lukios die vorbereitenden Handlungen zu dem sexuellen Akt unternimmt.
} 
subjektiven Eindruck, er könne hier nicht mehr ganz mithalten (22.4 illa vero, quotiens ei parcens nates recellebam, accedens toties nisu rabido et spinam prehendens meam adplicitiore nexu inhaerebat, ut hercules etiam deesse mihi aliquid ad supplendam eius libidinem crederem, nec Minotauri matrem frustra delectatam putarem adultero mugiente).

Im Griechischen haben wir also einen Esel, der sich groteskerweise von seiner sprichwörtlich Eseln in besonderem Maße nachgesagten - Potenz im Stich gelassen fühlt, und sich daher gewaltig anstrengen muß, sein mythisches Paradigma, den Stier der Pasiphae, erreichen zu können; dieser seiner eigenen Insuffizienz bewusste Esel (der in dem wiederholten Verkehr mit der reichen Frau einen schlimmen Missbrauch

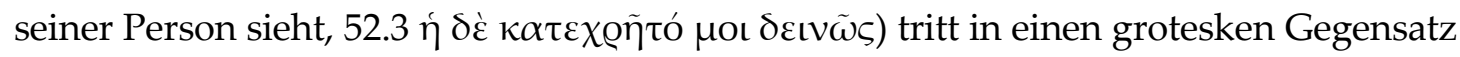
zum wieder vermenschlichten Lukios der Schlußszene, der aberwitzigerweise glaubt,

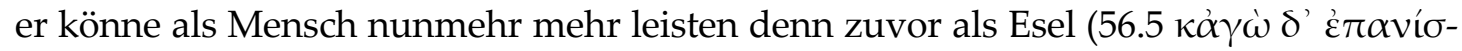

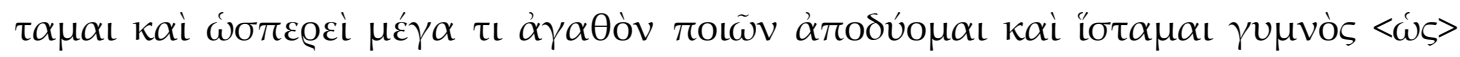

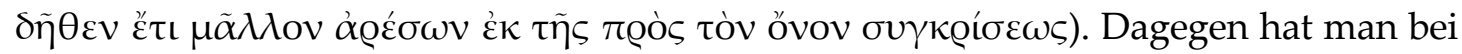
Apuleius einen Esel vor sich, der am Vollwert seiner sprichwörtlichen Qualitäten keinen Zweifel hat (vgl. auch 22.1 ex unguiculis perpruriscens); gerade vor diesem Hintergrund strahlt die Aussage, der - unzweifelhaft potente - Esel glaube, ihm fehle etwas, auf seine Liebhaberin ab, deren Wollust geradezu ins Unermessliche gesteigert wird, wenn ihr nicht einmal der sprichwörtlich potente Esel genügen zu können glaubt.

Es ergibt sich also der Befund, dass die Komik im Griechischen eher aufseiten des Lukios liegt, der seine relativen Fähigkeiten als Esel und als Mensch so grotesk falsch einschätzt, dagegen bei Apuleius die Komik sich eher in recht polemischer Form gegen die Frau richtet, die den objektiv potenten Esel einfach überstrapaziert.

Mit diesem Befund stimmt auch überein, dass der Esel bei Apuleius das Verhalten der Frau in sein allgemeines Bild von weiblicher Erotik einordnet. Dieser Esel hat ja vor allem in den zahllosen Ehebruch- und Hexenszenen, die er auf seinen verschiedenen Etappen miterlebte oder auch nur erzählt bekam (die dagegen im griechischen Vorbild wohl so gut wie keine Rolle spielten), reichlich Gelegenheit gehabt, sich ein einschlägiges Bild von weiblicher Wollust zu bilden. Dieses Bild erhält in der hier besprochenen Szene einen vorläufigen Höhepunkt, bevor es dann in der öffentlichen Zurschaustellung mit der ausführlich bezüglich ihrer Vorgeschichte dargestellten Schwerverbrecherin seine endgültige Kulmination erhält, die dann in der hilflosen Flucht des gepeinigten Esels und seinem an Isis gerichteten Erlösungswunsch resultiert. Man kann die Flucht von Apuleius' Esel geradezu auch als eine Flucht aus der heillosen Welt weiblicher Verfehlung in die Reinheit des Mystendaseins verstehen. ${ }^{7}$

Eine solche misogyne Tendenz ist dem griechischen Original, dessen Schlusspointe auf die groteske Fehleinschätzung des Lukios bezüglich seiner eigenen Fähigkeiten angelegt ist, natürlicherweise völlig fremd.

\footnotetext{
${ }^{7}$ Vgl. Müller-Reineke (2000: 193): „Der Esel Lucius findet im abschließenden 11. Buch der Metamorphosen Erlösung, indem er aller Leidenschaft und Neugierde entsagt und sich ganz der Göttin Isis weiht"; Schlam \& Finkelpearl (2000: 173): „The purity of the devotee stands in contrast to the lust dominant in the adventures", ferner a.a.O. 171-175 über "Sex and Venus” in den Metamorphosen des Apuleius. Die im Anschluß an I. Massey getroffene allgemeine Feststellung „Lucius is not devoted to sex" (a.a.O. 172), insofern er auch Fotis nur für seine Neugier instrumentalisiere, trifft auch für das aus dem Onos erschließbare griechische Original zu. Gerade vor diesem Hintergrund gewinnt der Besuch des Helden bei seiner früheren Liebhaberin am Ende der griechischen Fassung seine überraschende Wirkung.
} 
Und diese verschiedene tendenziöse Gestaltung der ganzen Beischlafszene schlägt sich konsequenterweise auch in der konträren Verwendung des mythischen PasiphaeParadigmas nieder: Der Esel der griechischen Version nimmt den Stier der Pasiphae als sein persönliches Vorbild bei dem Versuch, seine Verehrerin zufriedenzustellen

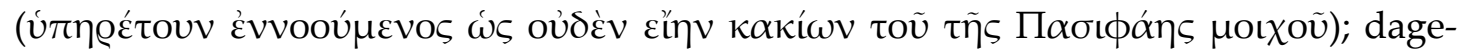
gen zieht der Esel des Apuleius aus dem stimulierenden Verhalten seiner Partnerin die Schlussfolgerung, dass er nunmehr die mythologische Figur der Pasiphae besser verstehen kann (ut ... nec Minotauri matrem frustra delectatam putarem adultero mugiente). ${ }^{8}$ Im Griechischen bezieht sich der mythologische Vergleich also auf die Bemühung des Esels, der sich mit dem Stier der Pasiphae vergleicht, und hat eine durchaus positive Wertigkeit, während bei Apuleius der Vergleich primär auf die Partnerin des Esels und ihre mythische Parallelfigur Pasiphae abzielt und diese Person gezielt diskreditiert, wie es ja nach der recht ambivalenten Anwendung des Pasiphae-Mythologems auf die Frau schlechthin im Eingang der ovidischen Ars amatoria durchaus naheliegt. Dies zeigt auch die vergleichende Charakterisierung der Verehrerin am Anfang der ganzen Episode, die in der griechischen Version keine Entsprechung hat (19.3): nec ullam vaesanae libidini medelam capiens ad instar asinariae Pasiphaae complexus meos ardenter exspectabat. Während im griechischen Vorbild der Esel durch den Stier-Vergleich sein geringes Potenzbewußtsein aufzuwerten versucht, rahmt er bei Apuleius mit den beiden Pasiphae-Vergleichen das abwertende Bild seiner Partnerin.

\section{Eine implizite Parallele: Der Esel Lucius und die Kuh Io bei Apuleius}

Bei Apuleius erhält der Esel Lucius nach diversen Episoden, in denen vor allem ehebrecherische und magiebewanderte Frauen im Mittelpunkt stehen, schließlich mit Hilfe der Göttin Isis seine menschliche Gestalt zurück. Besonders interessant ist die Art, in der sich die schließliche Rückverwandlung anbahnt:

Der eselgestaltige Lucius soll bei einer volksfestartigen Belustigung die Volksmenge unterhalten, indem er öffentlich mit einer weiteren menschlichen Frau Geschlechtsverkehr übt. Die Dame, die ihm hierzu als (nunmehr zweite) Partnerin bestimmt wird, ist eine abgeurteilte Schwerverbrecherin und Giftmischerin, deren Vorgeschichte zuvor in einem ausführlichen Exkurs (10.23-28) erzählt wurde. Als diese von einem Soldaten aus dem Kerker herbeigeführt wird (10.34 ecce quidam miles per mediam plateam dirigit cursum petiturus iam populo postulante illam de puplico carcere mulierem, quam dixi propter multiforme scelus bestis esse damnatam meisque praeclaris nuptiis destinatam), überlegt sich Lucius die möglichen Folgen eines solchen Beischlafs (at ego ... metu etiam mortis maxime cruciabar sic ipse mecum reputans) und ergreift schließlich in einem unbewachten Moment die Flucht (10.35 paulatim furtivum pedem proferens portam, quae proxima est, potitus iam cursu me celerrimo proripio). Nachdem er sechs Meilen im Galopp zurückgelegt hat (sexque totis passuum milibus perniciter confectis), erreicht er das Dorf Kegchreai bei Korinth ${ }^{9}$ und legt sich dort an einem einsamen Strandabschnitt in die Meeresbran-

\footnotetext{
${ }^{8}$ Eine ähnliche Beobachtung wird von Zimmerman (2000: 264 f.) auf die unpublizierte Dissertation von J. Seubers zurückgeführt.

${ }^{9}$ Eine besondere Ortssymbolik vermutet Mason 1971, der die Neueinführung dieses Schauplatzes bei Apuleius gegenüber dem griechischen Original hervorhebt und Korinth als „symbol of the secular life which Lucius/Apuleius rejected in the final Isis-book" (165) betrachtet. Vgl. jetzt auch Egelhaaf-Gaiser (2000: 68 ff.), die die Klimax von Kegchreai zu Rom als späterem Aufenthaltsort des Lucius betont.
} 
dung (prope ipsas fluctuum aspergines in quodam mollissimo harenae gremio lassum corpus porrectus refoveo). Noch in der Nacht richtet Lucius ein verzweifeltes Gebet an Isis (11.1 deam praepotentem lacrimoso vultu sic adprecabar).${ }^{10}$ Die Göttin erscheint in einem Traum Lucius persönlich (11.3 f.) und verheißt ihm die ersehnte Rettung in Form einer Rückverwandlung anläßlich ihres Festes (11.5 f. en adsum tuis commota, Luci, precibus etc.).

Der gesamte paraphrasierte Geschehensablauf läßt sich als eine Stukturimitation derjenigen Ereignisse verstehen, welche der Zurückverwandlung der verkuhten Io im ersten Metamorphosenbuch ${ }^{11}$ vorausgehen. Diese Kuh erfährt ihre letzte Prüfung, indem die eifersüchtige Götterkönigin Juno ihr eine Erinye vor Augen stellt (724-726 Protinus exarsit nec tempora distulit irael Horriferamque oculis animoque obiecit Erinyn/ Paelicis Argolicae). Auf diese Weise wird in Io eine panikartige Furcht erzeugt (726 f. stimulosque in pectore caecos/ Condidit). Diese Furcht führt zu einer rasenden Fluchtbewegung der Io durch die ganze Welt (727 et profugam per totum terruit orbem). Diese Flucht endet am Nil (728 Ultimus inmenso restabas, Nile, labori). Dort legt sich Io ans Ufer (729 f. Quem simulac tetigit, positisque in margine ripael Procubuit genibus) und richtet unter Tränen ein Gebet an ihren Geliebten Jupiter um Erlösung (730-733 resupinoque ardua collo,/ Quos potuit solos, tollens ad sidera vultus/ Et gemitu et lacrimis et luctisono mugitu/ Cum Iove visa queri finemque orare malorum). Dieser Bitte entspricht Jupiter mit einer ausdrücklichen Verheißung an Io (735-737 rogat "in" que "futurum/ Pone metus" inquit: "numquam tibi causa doloris/ Haec [sc. Iuno] erit," et Stygias iubet hoc audire paludes).

Sämtliche Details dieses Erzählablaufs (1. das Erscheinen einer furchtbaren Frauengestalt, 2. die dadurch ausgelöste Schreckreaktion der betroffenen Person, 3. die sich über einen weiten Raum erstreckende Flucht derselben, 4. die Ankunft an einem Gewässer, 5. die Niederlassung des erschöpften Flüchtlings an diesem Gewässer, 6. das Gebet um die erlösende Rückverwandlung an eine Gottheit, 7. die Epiphanie derselben Gottheit und 8. die zusagende Verheißung) stimmen exakt überein. Dabei sind trivialisierend-parodisierende Züge seitens der Nachbildung des Apuleius nicht zu verkennen: Für das weibliche Unterweltswesen tritt die kriminelle Giftmischerin ein, die Furcht des Ichs des Esels bezieht sich konkret darauf, dass die wilden Tiere, denen seine Partnerin strafweise ausgesetzt ist, auch über ihn herfallen könnten, und seine panische Flucht erstreckt sich nicht über die ganze Welt, sondern nur über die vergleichsweise lange Strecke von sechs Meilen bis zu einem - auch als Kultort der Isis offenbar nicht übermäßig bedeutsamen - korinthischen Vorort, dessen Strand das Nilufer im ovidischen Original vertritt.

Was diese offenkundige Imitation ovidischer Erzählstrukturen besonders bedeutsam macht, ist ihre Stellung im Ganzen der Metamorphosen des Apuleius. In dem griechischen Vorbild, welches sich aus dem pseudo-lukianischen Onos erschließen lässt, entkommt der Esel der letzten ihm drohenden erotischen Zumutung, indem er im äußersten Moment die ihn rettenden Rosen erblickt und sich damit auf dem Wege einer Rückverwandlung seiner Pflicht entzieht. Gerade mit der durch den Anblick der

\footnotetext{
${ }^{10}$ Aus der Tatsache, dass der Esel, nachdem er sich am Strand niedergelegt hat, plötzlich über die Gabe der Sprache verfügt, leitet E. Finkelpearl (2003) eine Verbindung zur Aesop-Vita ab, gemäß welcher dem Aesop im Schlaf von Isis die Gabe der Sprache geschenkt wurde.

${ }^{11}$ Zur bisherigen Forschung über die Beziehungen des Apuleius zu Ovid vgl. Schlam \& Finkelpearl 2000: 209-211, besonders die Arbeit von Müller-Reineke (2000). Die Nachwirkungen u.a. der ovidischen IsisEpisode bezüglich der Schilderung des eigentlichen Verwandlungsvorgangs untersucht Rosa Maria Lucifora (2004, besonders $222 \mathrm{ff}$.).
} 
Verbrecherin ausgelösten Flucht weicht Apuleius also von seinem griechischen Vorbild in entscheidender und wegweisender Weise ab.

Und gerade diese wegweisende Abweichung wird durch die besprochene signifikante Imitation der ovidischen Erzählstruktur markiert. Der Leser, der die Parallelitäten zwischen der Flucht des Esels und derjenigen der ovidischen Io erkennt, erhält bereits an der Grenze zum letzten Buch entscheidende Hinweise über das, was Apuleius vorhat: 1 . Er weicht ab von seiner bisherigen Vorlage, dem griechischen Eselsroman, was durch den hier punktuell besonders spürbaren Anschluß an die lateinischen Metamorphosen Ovids deutlich bekundet wird, und 2. er begibt sich in den kultisch-literarischen Bereich der griechisch-aegyptischen Gottheit Io-Isis. Die Flucht und Erlösung des künftigen Isis-Mysten Lucius wird in eine sinnhafte Beziehung zu den bei Ovid verewigten Erlebnissen seiner Göttin vor deren Apotheose gesetzt. Lucius wird so bereits, bevor er initiiert wird, intertextuell als ein Isis-Jünger gekennzeichnet, bzw. seine Eselsgestalt tritt in eine literarische Beziehung zur Kuhgestalt der Io. Wie seine Göttin muß Lucius die Erfahrung per aspera ad astra machen. Die besprochene Strukturimitation an der Grenze zum Schlußbuch markiert exakt den Übergang zwischen aspera und astra.

Prof. Dr. Thomas Gärtner

Institut für Altertumskunde

Universität zu Köln

E-mail: th-gaertner@gmx.de 


\section{BIBLIOGRAPHY}

Babo, Markus (2000) ,Mythologische Exempla als teleologische Elemente in den Metamorphosen des Apuleius.' - Maia 52, 485-496.

Bernsdorff, Hans (1997) ,Lukios als exclusus amator (zu [Luc.] asin. 56).' - Mnemosyne $50,35-44$.

Bohm, Robert Karl (1973) ,The Isis episode in Apuleius.' - CJ 68.3, 228-231.

Effe, Bernd (1976) ,Der missglückte Selbstmord des Aristomenes (Apul. Met. I, 14-17). Zur Romanparodie im griechischen Eselsroman.' - Hermes 104, 362-375.

Egelhaaf-Gaiser, Ulrike (2000) Kulträume im römischen Alltag: das Isisbuch des Apuleius und der Ort von Religion im kaiserzeitlichen Rom. (Potsdamer altertumswissenschaftliche Beiträge; 2.) Stuttgart: Steiner.

Finkelpearl, Ellen (2003) ,Lucius and Aesop gain a voice. Apuleius met. 11.1-2 and vita Aesopi 7.' - Panayotakis, Stelios; Zimmerman, Maaike; Keulen, Wytse (eds.), The ancient novel and beyond. (Mnemosyne Supplementum; 241.) Leiden etc: Brill, 37-51.

Harrison, Stephen J. (2000) Apuleius: a Latin sophist. Oxford: Oxford University Press.

Hindermann, Judith (2009) Der elegische Esel. Apuleius' Metamorphosen und Ovids Ars amatoria. (Studien zur klassischen Philologie; 162.) Frankfurt am Main etc: Lang.

Holzberg, Niklas (2006) Der antike Roman: eine Einführung. 3., überarb. Aufl. Darmstadt: Wissenschaftliche Buchgesellschaft.

Kussl, Rolf (1990), Die Metamorphosen des 'Lukios von Patrai'. Untersuchungen zur Phot. Bibl. 129.' - RhM 133, 379-388.

Lucifora, Rosa Maria (2004) ,Il racconto della metamorfosi dall'epica (di Ovidio) al romanzo (di Apuleio): 1'esegesi antica.' - Pretagonisti, Roberto; Dettori, Emanuele (eds.), La cultura ellenistica: l'opera letteraria e l'esegesi antica. (Quaderni dei seminari romani di cultura greca; 8.) Roma: Quasar, 213-230.

Mal-Maeder, Danielle van (1997) ,Lector, intende: laetaberis. The enigma of the last book of Apuleius' Metamorphoses.' - Groningen Colloquia on the Novel 8, 87-118.

Mason, Hugh J. (1971) ,Lucius at Corinth.' - Phoenix 25, 160-165.

Müller-Reineke, Hendrik (2000) Liebesbeziehungen in Ovids "Metamorphosen" und ihr Einfluss auf den Roman des Apuleius. 2. verb. Aufl. Göttingen: Hainholz.

Murgatroyd, Paul (2004) ,The ending of Apuleius' Metamorphoses.' - CQ 54, 319-321.

Schlam, Carl C.; Finkelpearl, Ellen (2000) ,A review of scholarship on Apuleius' Metamorphoses 1970-1998.' - Lustrum 42, 7-225.

Scobie, Alex (1978) ,The structure of Apuleius' Metamorphoses.' - Hijmans, Benjamin Lodewijk; Paardt, Rudolf Theodoor van der (eds.), Aspects of Apuleius' Golden ass. Groningen: Bouma, 43-61.

Töchterle, Karlheinz (1998) ,Apuleius als Isisjünger im Rahmen der Erzählstruktur seines Romans.' - Tschuggnall, Peter (ed.), Religion - Literatur - Künste: Aspekte eines Vergleichs. Anif/Salzburg: Müller-Speiser, 179-190.

Zimmerman, Maaike (2000) Apuleius Madaurensis Metamorphoses X. Text, introduction and commentary. Groningen: Forsten. 